\title{
The predictive value of urinary kidney injury molecular-1 for long-term graft function in kidney transplant patients: a prospective study
}

\author{
Minyan Zhu ${ }^{1 \# \wedge}$, Zhejun Chen ${ }^{1 \#}$, Yuehan Wei ${ }^{1}$, Yanhong Yuan ${ }^{1}$, Liang Ying ${ }^{2}$, Hang Zhou ${ }^{1}$, Xiajing Che ${ }^{1}$, \\ Min Fang Zhang ${ }^{1}$, Zhaohui $\mathrm{Ni}^{1}$, Ming Zhang ${ }^{2}$, Shan Mou ${ }^{1 \wedge}$ \\ ${ }^{1}$ Department of Nephrology, Molecular Cell Laboratory for Kidney Disease, Renji Hospital, School of Medicine, Shanghai Jiao Tong University, \\ Shanghai, China; ${ }^{2}$ Transplantation Center of Renji Hospital, School of Medicine, Shanghai Jiao Tong University, Shanghai, China \\ Contributions: (I) Conception and design: S Mou, M Zhang; (II) Administrative support: Z Ni, S Mou, M Zhang; (III) Provision of study materials or \\ patients: L Ying, X Che, MF Zhang, Z Chen; (IV) Collection and assembly of data: M Zhu, Z Chen, Y Wei; (V) Data analysis and interpretation: M \\ Zhu, Y Yuan, H Zhou; (VI) Manuscript writing: All authors; (VII) Final approval of manuscript: All authors. \\ \#These authors contributed equally to this work. \\ Correspondence to: Shan Mou. Department of Nephrology, Molecular Cell Laboratory for Kidney Disease, Renji Hospital, School of Medicine, \\ Shanghai Jiao Tong University, Shanghai 200127, China. Email: shan_mou@shsmu.edu.cn; Ming Zhang. Transplantation Center of Renji Hospital, \\ School of Medicine, Shanghai Jiao Tong University, 160 Pujian Road, Shanghai 200127, China. Email: drmingzhang@126.com.
}

\begin{abstract}
Background: Monitoring allograft function during the early stages is crucial, and therefore requires biomarkers more sensitive than serum creatinine (Scr). Kidney injury molecular-1 (KIM-1) is a potent biomarker; however, disparities exist in the literature concerning its predictive value in allograft function. Therefore, this study aimed to evaluate its predictive value for the long-term prognosis of kidney transplantation patients.
\end{abstract}

Methods: A prospective study with a cohort comprising 160 patients scheduled for kidney transplantation was conducted to evaluate the predictive power of urinary KIM-1 (uKIM-1) and other renal ischemiareperfusion biomarkers including urinary L-type fatty acid binding protein (uL-FABP), urinary N-acetyl$\beta-\mathrm{D}$ glucosaminidase (uNAG), and urinary neutrophil gelatinase-related lipoprotein (uNGAL) for allograft prognosis.

Results: One hundred and forty kidney recipients who were admitted to our hospital between September 2014 and December 2017 with a median follow-up of 30.3 months were included. Thirty-seven recipients had functional delayed graft function (fDGF) in the first week post transplantation, and 42 recipients had progressed to allograft dysfunction [estimated glomerular filtration rate (eGFR) $<60 \mathrm{~mL} / \mathrm{min} / 1.73 \mathrm{~m}^{2}$ ] by the end of the study, while nine recipients deteriorated into allograft loss (defined by the initiation of dialysis). The levels of uKIM-1 in the fDGF group were higher than those in the immediate graft function (IGF) recipients $(\mathrm{P}<0.05)$ at 0 hour post transplantation [5.885 (4.420-7.913) vs. 4.605 (3.417-5.653) ng/mmol], and on the first day post transplantation [5.569 (4.181-6.722) vs. $4.002(3.222-6.488) \mathrm{ng} / \mathrm{mmol}]$. The levels of uL-FABP in the fDGF group were also higher than those in the IGF group at 0 hour post transplantation $(89.818 \pm 39.332$ vs. $69.187 \pm 37.926 \mu \mathrm{g} / \mathrm{mmol})$ and on the third day post transplantation $[77.835(60.368-$ $100.678)$ vs. $66.841(28.815-89.783) \mu \mathrm{g} / \mathrm{mmol}]$. Multivariate Cox regression analysis demonstrated that recipients with higher uKIM-1 levels on the first day post transplantation had a $23.5 \%$ increase in the risk of developing fDGF and a $27.3 \%$ increase in the risk of prolonged renal allograft dysfunction.

Conclusions: uKIM-1 on the first day post transplantation can predict short-term graft function and is a potent biomarker for the long-term prognosis of graft function.

^ ORCID: Minyan Zhu, 0000-0002-1868-3896; Shan Mou, 0000-0003-4160-1681. 
Keywords: Biomarker; kidney transplantation; delayed graft function (DGF); predictive value; graft survival

Submitted Mar 05, 2020. Accepted for publication Nov 08, 2020.

doi: $10.21037 /$ atm-20-2215a

View this article at: http://dx.doi.org/10.21037/atm-20-2215a

\section{Introduction}

Despite surgical techniques and immunotherapy having progressed rapidly in recent years, the incidence of delayed graft function (DGF) and the occurrence of longterm adverse events after kidney transplantation are still increasing $(1,2)$. One of the reasons for this is the expansion of the donor pool through donation after cardiac death (DCD) in the last few decades, accompanied by the increased incidences of DGF and long-term graft loss caused by ischemia-reperfusion injury $(3,4)$. Elderly donors, prolonged cold ischemia time, and recipient characteristics [including age, human leukocyte antigen (HLA) matches, panel-reactive antibody (PRA) percentages] have also been reported to be factors affecting the incidences of DGF and long-term graft survival $(2,5)$. Thus, monitoring allograft function at an early stage is of paramount importance. The two clinical indicators that currently exist are not adequate for this purpose (6); serum creatinine (Scr) shows inadequate sensitivity, while obtaining surveillance allograft biopsy is invasive. Therefore, there is growing interest in exploring blood and urine biomarkers for predicting the prognosis of kidney transplantation patients.

To predict both short- and long-term prognosis of patients with renal transplantation, non-invasive and diagnostic urinary or serum biomarkers are needed for early detection. The existing method for assessing allograft function requires invasive renal puncture, which can cause hemorrhage and other complications. Studies focused on kidney tubular injury-related proteins, namely neutrophil gelatinase-related lipoprotein (NGAL), kidney injury molecule-1 (KIM-1), and interleukin-18 (IL-18), as potential markers for monitoring DGF are under investigation (6-8). Nevertheless, these biomarker candidates still lack clinical validation or positive predictive value for long-term allograft function (7). Moreover, NGAL and IL-18 are also produced by immune cells during urinary tract infections and sepsis. The confounding effect of infections or sepsis on the predictive value of NGAL and IL-18 for allograft prognosis has not been clarified in those studies.

KIM-1 is a transmembrane immunoglobulin that is not detectable in healthy kidneys. It is dramatically upregulated in damaged tubular epithelial cells in multiple types of kidney injury. Disparities exist within the literature on the predictive value of KIM-1 in allograft function. For instance, in a cohort study of 145 renal transplantation recipients, KIM-1 was reported to be a potent predictor of transplantation failure (9); however, another study found that KIM-1 could not effectively predict DGF (10).

Therefore, to determine the predictive value of KIM1 for both the short-term and long-term prognosis of kidney transplantation, we conducted a cohort study of renal transplantation recipients. We present the following article in accordance with the STROBE reporting checklist (available at http://dx.doi.org/10.21037/atm-20-2215a).

\section{Methods}

\section{Study subjects}

This study was a prospective, single-center cohort study from September 2014 to December 2017. The inclusion criteria were as follows: (I) end-stage renal disease (ESRD) patients aged 18-65 years old who were scheduled to receive kidney transplantation through DCD in the hospital within 3 days who (II) had an HLA mismatch (ABDR) at no more than 3 sites, and (III) had received anti-thymocyte globulin (ATG) as induction therapy, and tacrolimus with mycophenolate mofetil as the maintenance immunosuppression regimen. The exclusion criteria were as follows: (I) recipients who had received transplantation before or who had been infected with human immunodeficiency virus (HIV) or the hepatitis C virus; (II) recipients who were infected or had sepsis during follow-up.

The patients were followed up every week within the first month post transplantation, every month within the first year post transplantation, and every 3 months afterwards. Each enrolled patient had been followed up for at least 1 year. With reference to Boom et al. (11), functional delayed graft function (fDGF) was defined as a recovery of Scr of $<70 \%$ in the first week after transplantation, regardless of dialysis. Conversely, immediate graft function (IGF) was characterized by a recovery of Scr of $>70 \%$ in the first week after transplantation 
$(10,12)$. The study was conducted in accordance with the Declaration of Helsinki (as revised in 2013). The study was approved by the Ethics Committee of Renji Hospital, School of Medicine, Shanghai Jiao Tong University (14140903200). All patients provided informed consent.

\section{Endpoint definitions}

The primary endpoint for the analysis was allograft loss (marked by the initiation of dialysis). Time to event was calculated from enrollment to either allograft loss or the end of the study (13). The secondary endpoint was graft dysfunction [estimated glomerular filtration rate (eGFR) $\left.<60 \mathrm{~mL} / \mathrm{min} / 1.73 \mathrm{~m}^{2}\right]$.

\section{Sampling}

Fresh blood samples were collected from all patients within the 1 week before transplantation; specifically, immediately after the perfusion of the donor kidney ( 0 hour), and at 24 and 72 hours after transplantation. Urine samples were obtained before transplantation if the patient was able to urinate, and collected from the bag and catheter of each patient after the perfusion of the donor kidney during transplantation, and at 24 and 72 hours after transplantation. All samples were submitted to the biochemical laboratory for analysis shortly after collection. The rate of centrifugation was $3,500 \times \mathrm{g}$ for $15 \mathrm{~min}$ for fresh urine $(10 \mathrm{~mL})$ and serum $(500 \mu \mathrm{L})$. The supernatant was then transferred to Eppendorf tubes and stored at $-80{ }^{\circ} \mathrm{C}$ until further analysis.

\section{Biomarker measurement}

The levels of KIM-1, L-type fatty acid binding protein (L-FABP), NGAL, and N-acetyl- $\beta$-D glucosaminidase (NAG) in the patients' serum and urine were examined using enzyme-linked immunosorbent assay (ELISA) kits from American R\&D Corporation (Minneapolis, MN, USA). A standard curve was generated to calculate the concentration of the samples. The results were corrected through synchronous normalization to urinary creatinine (uCr), which was measured by sarcosine oxidase method $(14,15)$.

\section{Other measurements}

Patient characteristics assessed at enrollment included demographic features (age and sex), physical examination findings [body mass index (BMI)], medical history (hypertension and diabetes mellitus), and transplantation features (duration of dialysis and mode of dialysis before transplantation). Routine pathology tests were also performed 1 day before transplantation, and included the following markers: Scr, hemoglobin, serum albumin, serum globulin, white blood cells, serum potassium, serum sodium, serum bicarbonate, pre-albumin, glutamate pyruvic transaminase, aspartate aminotransferase, $\gamma$-glutamyl transpeptidase, alkaline phosphatase, serum total cholesterol, serum triglyceride, serum high density lipoprotein, serum low-density lipoprotein, serum calcium ion, serum phosphorus, and immunoreactive serum parathyroid hormone.

Scr was measured by sarcosine oxidase method pretransplantation, and at 0 hour, 1, 3, 5, 7, 10, 14, 21 days, every 1 month within the first year post transplantation, and every 3 months thereafter (4). eGFR was calculated using the modification of diet in renal disease (MDRD) formula: $\mathrm{eGFR}=186 \times(\mathrm{Scr} / 88.4)^{-1.154} \times \mathrm{age}^{-0.203}(\times 0.742$, female $)$ $(16,17)$. BMI was calculated by weight $(\mathrm{kg}) /$ height $(\mathrm{m})^{2}$. Medical history was self-reported at admission.

\section{Statistical analysis}

Statistical analysis was performed with IBM SPSS Statistics (version 25, SPSS Inc., Chicago, IL, USA), GraphPad Prism (version 8.0, GraphPad Software, San Diego, CA, USA), and survival package in $\mathrm{R}$, version 6.0.1. Before analysis, all data were tested for normal distribution. Student's $t$-test was applied to parametric continuous variables, the Mann-Whitney $\mathrm{U}$ test was performed for non-parametric data, and a chi-squared test was used for categorical variables. Comparisons between multiple groups were performed using analysis of variance (ANOVA) for parametric variables and the Kruskal-Wallis test for nonparametric variables. Kaplan-Meier survival analysis was carried out to compare allograft survival between the fDGF and IGF groups, together with the log-rank test for calculation. Receiver operating characteristic (ROC) analysis was performed to determine the biomarker thresholds that were best associated with fDGF development, and Spearman's correlation analysis was used to measure the degree of association between variables. Univariate and multivariate linear regression analyses were performed to examine the relationships between fDGF and independent variables. Univariate and multivariate Cox 


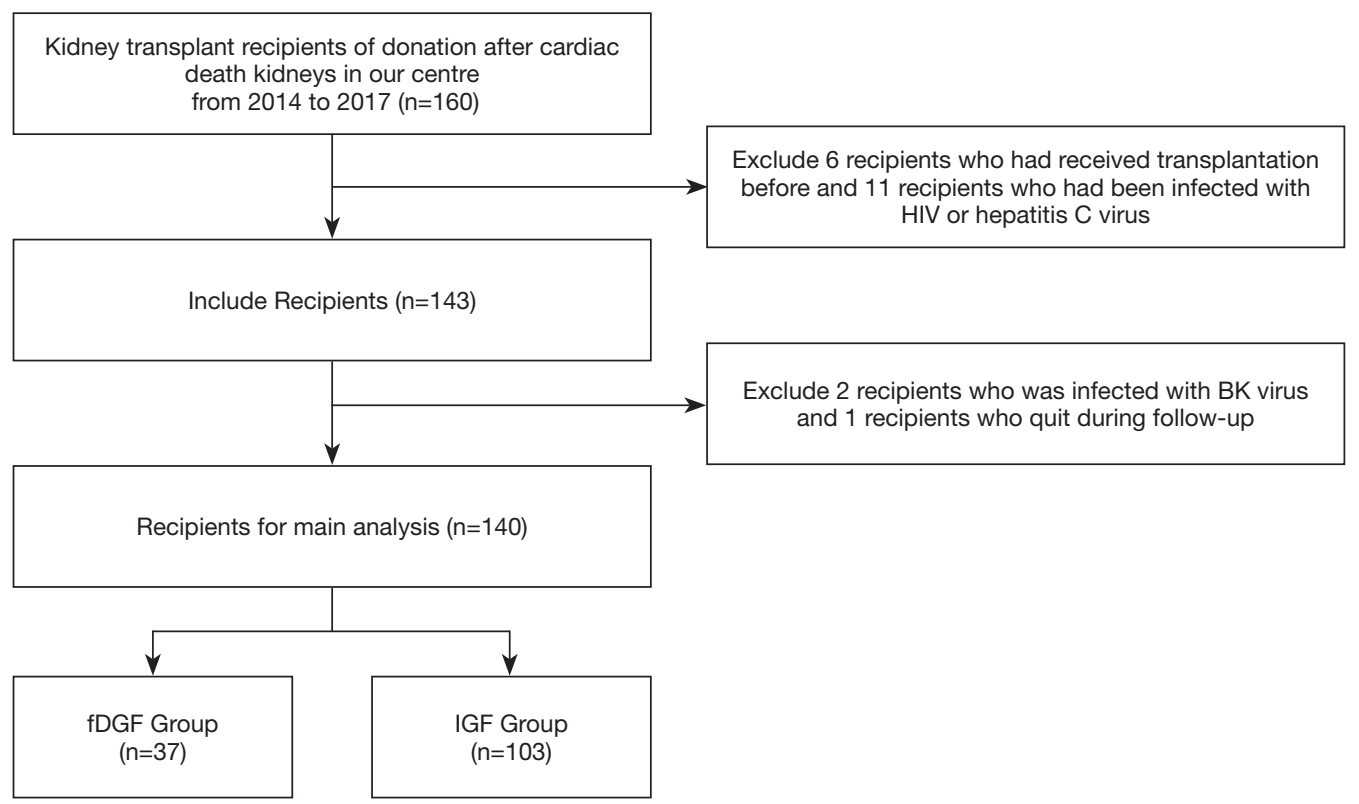

Figure 1 Flow diagram of the study design. fDGF, functional delayed graft function; IGF, immediate graft function.

regression analyses were performed to assess the hazard ratios (HRs) and 95\% confidence intervals (CIs). For all tests, statistical significance was considered as a two-sided $\mathrm{P}$ value of $\leq 0.05$. Normally distributed data were expressed as mean \pm standard deviation (SD), non-normally distributed data were expressed as median (interquartile range), and categorical data were expressed as frequencies (percentages).

\section{Results}

\section{Patient characteristics}

A total of 160 kidney transplantation recipients through DCD were included in this study between September 2014 to December 2017. A total of 17 transplant recipients who had HIV or hepatitis C virus infection were excluded. Subsequently, 143 patients were followed up for a median duration of 30.3 months (IQR, 9.18-44.25 months) after transplantation. Three recipients were excluded during follow-up due to BK virus infection or loss to follow-up. Finally, a total of 140 patients were enrolled for the final analysis (Figure 1).

At baseline, the median age of the 140 enrolled recipients was 41.0 years old (32.0-50.0 years old). A total of 37 recipients progressed to fDGF during the first week after transplantation; 7 of these fDGF cases were elicited by allograft rejection and another 3 by surgical reasons.
A total of 3 recipients in the IGF group had allograft rejection during the follow-up after the first week post transplantation. There were no statistically significant differences between fDGF and IGF recipients in terms of HLA mismatches, hot and cold ischemia time, creatinine clearance rate, donor age, or medical history before transplantation (Table $1 \&$ Tables S1-S3).

\section{Increased long-term graft dysfunction in patients with fDGF}

A total of 42 recipients exhibited impaired graft function (eGFR $\left.\leq 60 \mathrm{~mL} / \mathrm{min} / 1.73 \mathrm{~m}^{2}\right)(17,45.90 \%$ in fDGF group vs. $25,24.20 \%$ in IGF group, $\mathrm{P}=0.004)$ during the last follow-up visit (Table S3). Nine recipients deteriorated into allograft loss by the end of this study (Table S4). The fDGF group included $7(18.9 \%)$ allograft loss recipients, which was more than the $2(1.9 \%)$ in the IGF group $(\mathrm{P}=0.000)$. Among the 9 recipients who deteriorated into allograft loss, 3 died during dialysis, due to allograft rejection, infection, and cardiac arrest, respectively, although this was not statistically significant.

\section{Comparison of biomarkers between recipients with and without fDGF}

Both urinary KIM-1 (uKIM-1)/uCr and urinary L-FABP 
Table 1 Characteristics of transplant recipients at baseline

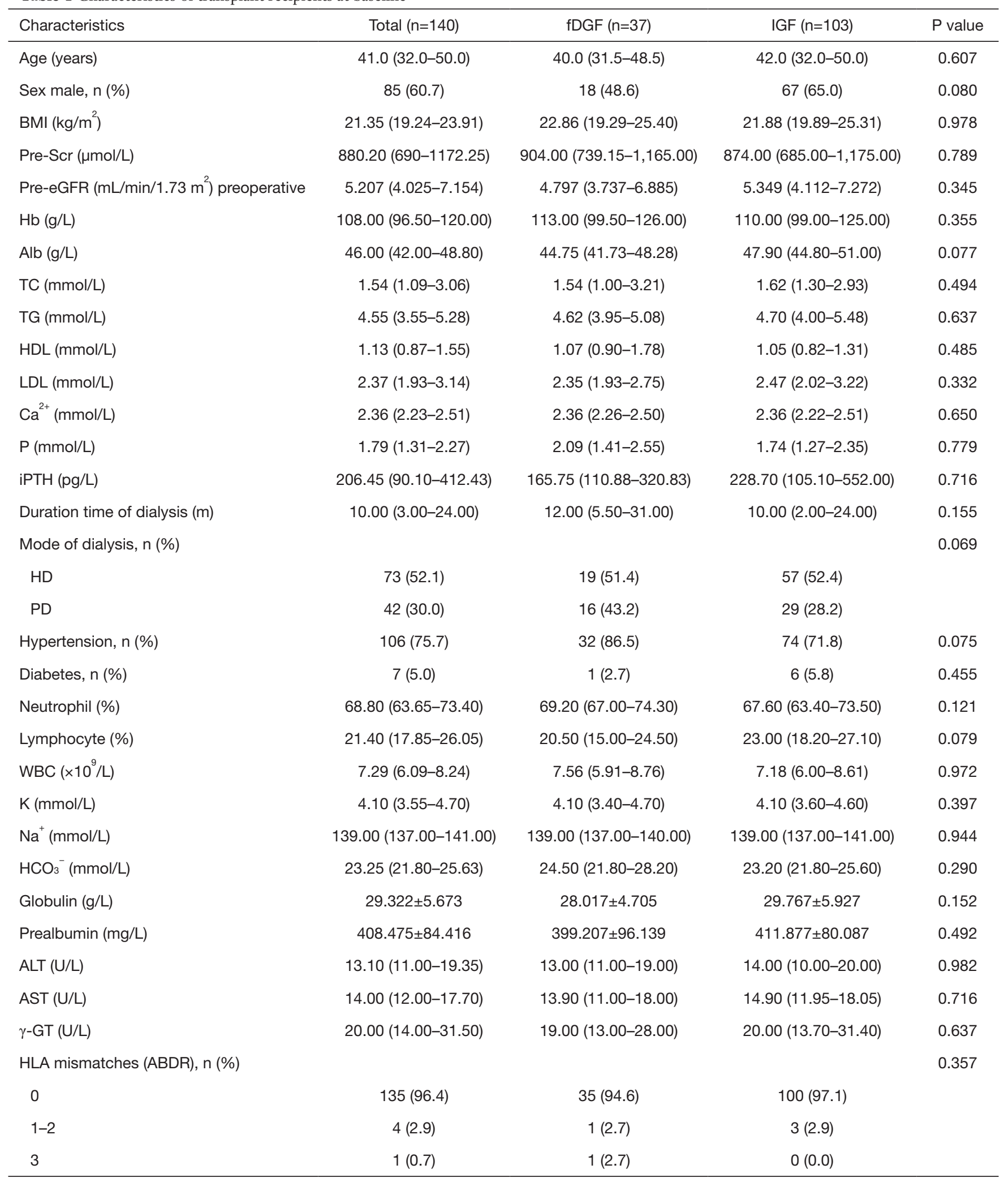

Table 1 (continued) 
Table 1 (continued)

\begin{tabular}{|c|c|c|c|c|}
\hline Characteristics & Total $(n=140)$ & fDGF $(n=37)$ & IGF $(n=103)$ & $P$ value \\
\hline Cold ischemia time (hours) & $4.60(2.50-5.10)$ & $4.30(2.25-4.95)$ & $4.70(3.40-5.10)$ & 0.067 \\
\hline
\end{tabular}

Data that conform to a normal distribution are represented by the mean \pm standard deviation (SD), and non-normally distributed data are represented by the median (interquartile range). Frequency of occurrence of the event is expressed as the frequency (percentage). fDGF, functional delayed graft function; IGF, immediate graft function; BMI, body mass index; pre-Scr, pre-operative serum creatinine; pre-eGFR, pre-operative estimated glomerular filtration rate; Hb, hemoglobin; Alb, serum albumin; TC, serum total cholesterol; TG, serum triglyceride; HDL, high-density lipoprotein cholesterol; LDL, low-density lipoprotein cholesterol; $\mathrm{Ca}^{2+}$, serum total calcium; P, serum phosphorus; iPTH, immunoreactive serum parathyroid hormone; HD, hemodialysis; PD, peritoneal dialysis; WBC, white blood cells; K, serum potassium; $\mathrm{Na}^{+}$, serum sodium; $\mathrm{HCO}_{3}{ }^{-}$, serum bicarbonate; ALT, glutamate pyruvic transaminase; AST, aspartate aminotransferase; $\gamma$-GT, $\gamma$-glutamyl transpeptidase; ALP, alkaline phosphatase; HLA, human leukocyte antigen.

(uL-FABP)/uCr were higher in the fDGF group than in the IGF group immediately after transplantation, at 0 hour [uKIM-1/uCr: 5.885 (4.420-7.913) vs. 4.605 (3.417$5.653) \mathrm{ng} / \mathrm{mmol}, \mathrm{P}<0.05 ; \mathrm{uL}-\mathrm{FABP} / \mathrm{uCr}: 89.818 \pm 39.332$ vs. $69.187 \pm 37.926 \mu \mathrm{g} / \mathrm{mmol}, \mathrm{P}<0.05$; Figure 2, Table 2]. On the first day after transplantation, only uKIM- $1 / \mathrm{uCr}$ was significantly different between the two groups [5.569 (4.181-6.722) vs. 4.002 (3.222-6.488) ng/mmol, $\mathrm{P}<0.05]$. However, on the third day after transplantation, only $\mathrm{uL}-\mathrm{FABP} / \mathrm{uCr}$ was significantly different between the two groups [77.835 (60.368-100.678) vs. 66.841 (28.815 -89.783) $\mu \mathrm{g} / \mathrm{mmol}, \mathrm{P}<0.05]$. Other biomarkers did not show significant differences between the two groups. The levels of serum biomarkers were also tested, but no differences were observed (Table S5).

\section{Correlation between uKIM-1 and fDGF}

Correlation between uKIM-1 and fDGF was shown in Figure 3. On both the seventh day post transplantation and at the last follow-up visit, Scr was significantly higher and eGFR significantly lower in the fDGF group than in the IGF group (Figure $3 A, B)$. Spearman's correlation analysis indicated that uKIM-1 on the first day post-surgery was positively correlated with eGFR on the seventh day postsurgery $\left(\mathrm{r}^{2}=0.1092, \mathrm{P}<0.0001\right)$ and at the last visit $\left(\mathrm{r}^{2}=0.1045\right.$, $\mathrm{P}<0.0001$ ), respectively (Figure $3 E, F$ ). The correlation between uKIM-1 (first day post-surgery) and Scr started from the third day post-surgery $(\mathrm{r}=0.256, \mathrm{P}<0.05$, Table S6). Univariate and multivariate cox regression analyses demonstrated a $23.5 \%$ increase in the risk for fDGF for each $1 \mathrm{ng} / \mathrm{mmol}$ increase in uKIM-1 on the first day post-surgery while $\mathrm{uL}-\mathrm{FABP}$ demonstrate no statistical significance in the effect for fDGF in the multivariate cox regression analyses
(Figure S1).

\section{Assessment of short-term and long-term graft function on the basis of uKIM-1 levels on the first day post transplantation}

Figure S2 displays the ROC curves of uKIM-1/uCr at multiple timepoints for predicting the diagnosis of fDGF. On the first day post-surgery, uKIM-1/uCr exhibited a sensitivity of $81.10 \%$, which was the highest among all biomarkers, together with a specificity of $52.40 \%$ for fDGF. The combination of uKIM-/uCr and Scr on the first day post-surgery had the second highest area under curve (AUC) for fDGF at 0.761 (95\% CI, 0.664-0.857).

By reclassifying recipients into the KIM-1-high group (uKIM- $1 / \mathrm{uCr} \geq$ cutoff value for fDGF, $4.08 \mu \mathrm{g} / \mathrm{mmol}$ ) or the KIM-1-low group (uKIM-1/uCr < cutoff value for fDGF, $4.08 \mu \mathrm{g} / \mathrm{mmol}$ ), the median Scr were higher, and eGFR were lower in the uKIM-1-high group than in the uKIM-1-low group on the seventh day post transplantation and during the last follow-up visit (Figure 3C,D).

\section{uKIM-1 can predict long-term graft prognosis}

To determine whether uKIM-1 is related to long-term allograft prognosis, recipients who once presented with increased Scr due to surgical reasons, allograft rejection, infection, or cardiac arrest during the follow-up period were excluded. Ultimately, 125 recipients were included in the Kaplan-Meier analysis. The Kaplan-Meier plots indicated that recipients in the KIM-1-low group had a higher graft function survival rate $\left(e G F R>60 \mathrm{~mL} / \mathrm{min} / 1.73 \mathrm{~m}^{2}\right)$ than those in the KIM-1-high group (log-rank test, $\mathrm{P}<0.0001$; Figure 4). 

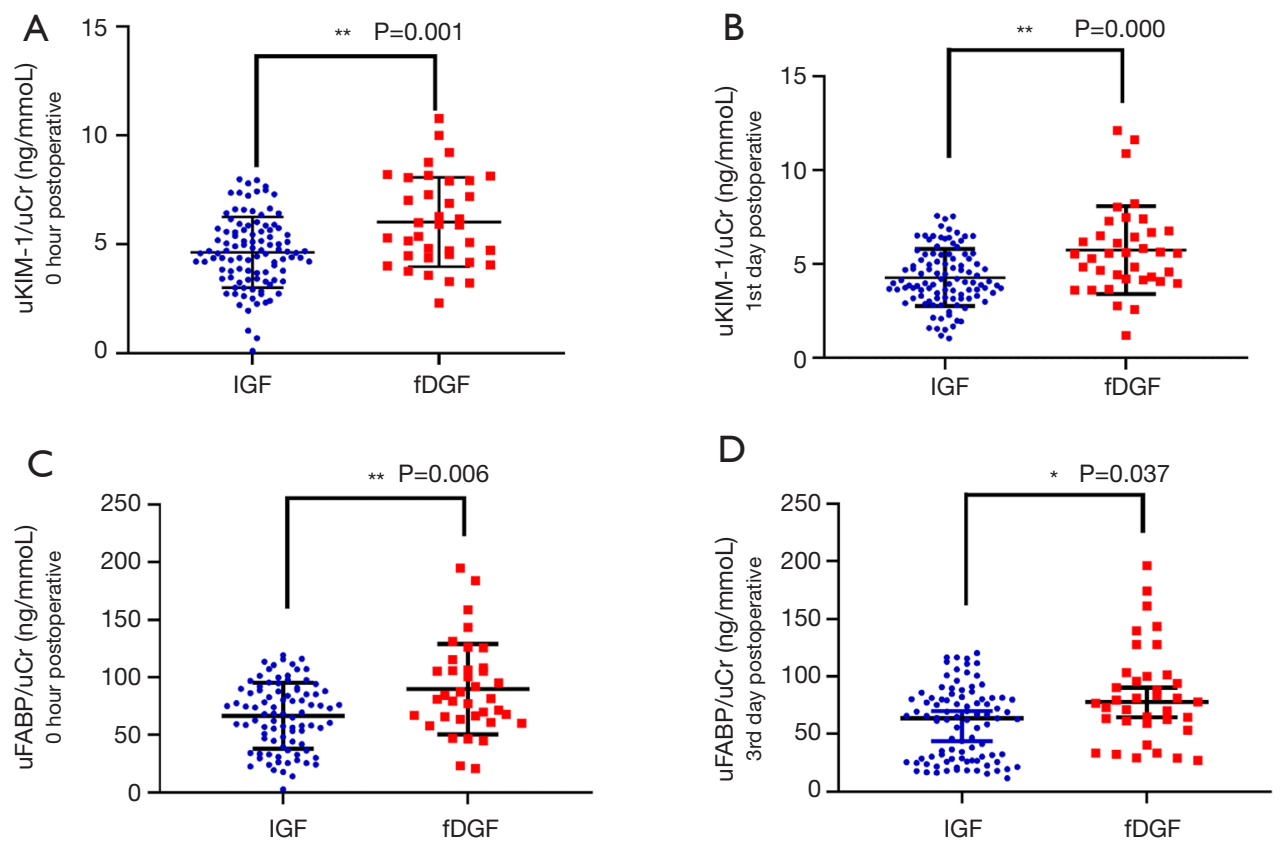

Figure 2 Levels of uKIM-1/uCr (at 0 hour and day 1 post transplantation) and uL-FABP/uCr (at 0 hour and day 3 post-transplantation) in the fDGF group were both significantly higher than those in the IGF group after kidney transplantation. (A) uKIM-1/uCr expression at 0 hour post-surgery in the fDGF and the IGF group; (B) uKIM-1/uCr expression on day 1 post-surgery in the fDGF and the IGF group; (C) $\mathrm{uL}-\mathrm{FABP} / \mathrm{uCr}$ expression at 0 hour post-surgery in the fDGF and the IGF group; (D) uL-FABP/uCr expression on day 3 post-surgery in the fDGF and the IGF group. *, $\mathrm{P}<0.05$; **, $\mathrm{P}<0.01$. fDGF, functional delayed graft function; IGF, immediate graft function; uKIM-1, urinary kidney injury molecular 1; uL-FABP, urinary L-type fatty acid binding protein; uCr, urinary creatinine; uKIM-1/uCr, the ratio of uKIM-1 to $\mathrm{uCr}$; $\mathrm{uL}-\mathrm{FABP} / \mathrm{uCr}$, the ratio of uL-FABP to $\mathrm{uCr}$.

ROC curves revealed that uKIM- $1 / \mathrm{uCr}$ on the first day post-surgery had a sensitivity of $90.00 \%$ and a specificity of $51.60 \%$ for diagnosing long-term graft dysfunction (eGFR $<60 \mathrm{~mL} / \mathrm{min} / 1.73 \mathrm{~m}^{2}$, Figure 5 ). Univariate and multivariate Cox regression analyses demonstrated that uKIM-1 on the first day post-surgery increased the risk of developing allograft dysfunction by $27.3 \%$ in for each $1 \mathrm{ng} / \mathrm{mmol}$ increase (Figure 6).

\section{Discussion}

DGF is a critical risk factor for long-term allograft function $(11,18)$. Despite controversy over its definition, DGF fundamentally refers to the absence of allograft function following kidney transplantation $(11,19,20)$. An increasing number of studies have demonstrated that 30-day readmission, poorer graft function, acute rejection, allograft loss, and death occur more frequently in patients with DGF compared to non-DGF patients (21-23). Acute tubular necrosis is an important marker of the deterioration stage towards DGF (24); however, its prognostic significance for long-term graft outcome remains unclear. The broadening criteria for DCD kidney donors accounts for the growing incidence of DGF (21). Hence, biomarkers for the early detection of DGF are urgently needed. In this study, to consider the impact of slow graft function, we applied the definition of fDGF as a decrease of Scr of $<70 \%$ in the first week after transplantation, regardless of dialysis, with reference to Boom et al. and Hall et al. $(6,11)$.

During a median follow-up of 30.3 months, we discovered that high concentrations of uKIM-1 were strongly associated with fDGF and future allograft loss, while other acute kidney injury (AKI) biomarkers including L-FABP, NGAL, and NAG were not. These associations were independent of $\mathrm{uCr}$, Scr, and acute allograft rejection situations where immunity might be a confounding factor. These results provide insights for graft function monitoring of patients after transplantation, as well as a foundation for future studies in this field.

In our previous work, we studied the diagnostic value 
Table 2 Comparison of urinary biomarker levels between fDGF group and IGF group

\begin{tabular}{|c|c|c|c|c|c|}
\hline Biomarker & Time after transplant & Total $(n=140)$ & fDGF $(n=37)$ & IGF $(n=103)$ & $P$ value \\
\hline \multirow{2}{*}{$\begin{array}{l}\text { uKIM-1/uCr } \\
\text { (ng/mmol) }\end{array}$} & 1st day postoperative & $4.452(3.602-5.705)$ & $5.569(4.181-6.722)$ & $4.002(3.222-6.488)$ & $0.000^{\star \star}$ \\
\hline & 3rd day postoperative & $4.099(2.241-6.5913)$ & $4.362(3.501-6.950)$ & 3.696 (1.879-6.547) & 0.175 \\
\hline \multirow{2}{*}{$\begin{array}{l}\mathrm{uL}-\mathrm{FABP} / \mathrm{uCr} \\
(\mu \mathrm{g} / \mathrm{mmol})\end{array}$} & 0 hour postoperative & $74.639 \pm 39.237$ & $89.818 \pm 39.332$ & $69.187 \pm 37.926$ & $0.006^{\star \star}$ \\
\hline & 3rd day postoperative & 69.705 (32.723-92.533) & $77.835(60.368-100.678)$ & $66.841(28.815-89.783)$ & $0.037^{*}$ \\
\hline \multirow{3}{*}{$\begin{array}{l}\text { uNGAL/uCr } \\
\text { (ng/mmol) }\end{array}$} & 0 hour postoperative & $0.720(0.500-1.483)$ & $0.640(0.440-0.915)$ & $0.760(0.550-1.540)$ & 0.060 \\
\hline & 1st day postoperative & $0.740(0.513-1.268)$ & $0.700(0.500-0.895)$ & $0.820(0.510-1.390)$ & 0.147 \\
\hline & 3rd day postoperative & $0.775(0.500-1.275)$ & $0.610(0.510-1.140)$ & $0.810(0.480-1.350)$ & 0.357 \\
\hline $\begin{array}{l}\text { uNAG/uCr } \\
\text { (ng/mmol) }\end{array}$ & 3rd day postoperative & $12.125(7.248-1.155)$ & $13.341(11.142-17.158)$ & $11.554(6.453-17.237)$ & 0.083 \\
\hline
\end{tabular}

Data that conform to a normal distribution are represented by the mean \pm standard deviation (SD), and non-normally distributed data are represented by the median (interquartile range). ${ }^{*}, \mathrm{P}<0.05$; ${ }^{*}, \mathrm{P}<0.01$. fDGF, functional delayed graft function; IGF, immediate graft function; uKIM-1, urinary kidney injury molecular 1; uL-FABP, urinary fatty acid-binding protein; uCr, urinary creatinine; uNGAL, urinary neutrophil gelatinase-related lipoprotein; uNAG, N-acetyl- $\beta-D$ glucosaminidase; uKIM-1/uCr, the ratio of uKIM-1 to $u C r$; uL-FABP/uCr, the ratio of $u L-F A B P$ to $u C r ; ~ u N G A L / u C r$, the ratio of uNGAL to $u C r$; $u N A G / u C r$, the ratio of uNAG to $u C r$.

of novel and non-invasive markers for AKI (25-27); in the current study, we extended our investigation to renal transplantation. KIM-1 has diverse characteristics that make it an ideal biomarker for the prediction of graft function. Specifically, as a transmembrane protein, KIM1 is significantly upregulated in urine by injured proximal tubular cells after various types of kidney injury, but it remains undetectable in healthy kidneys (28). Serum KIM1 level testing revealed no difference between the fDGF and IGF groups, supporting KIM-1 as an independent urinary tubular injury marker. Also, the existence of an immunoglobulin and mucin domain allows KIM1 to participate in innate immunity downregulation by mediating epithelial cell phagocytosis of apoptotic cells, which subsequently reduces inflammation and innate immunity $(29,30)$. Furthermore, KIM-1 is also essential in maladaptive repair between AKI and progressive chronic kidney disease (CKD) by promoting monocyte chemotactic protein-1 (MCP-1)-dependent kidney fibrosis (31). Additionally, KIM-1 exhibits sensitivity-enhanced responses to AKI compared to the traditional marker Scr $(32,33)$, making it an ideal candidate as an early biomarker of graft function.

Our previous work examined the relationship between
AKI and CKD. In a case-control study of 201 participants with AKI, we observed that 98 patients did not recover renal function during 1 year of follow-up, whereas $50 \%$ of patients presented with renal function deterioration. A higher level of uKIM-1 was associated with an elevated risk of renal function deterioration (HR, 1.018; 95\% CI, 1.004-1.031) (16). Our studies in early and obstructive nephropathy demonstrated similar results $(26,34)$.

Few prior studies have evaluated the association between uKIM-1 and the long-term outcomes of kidney transplantation patients. While Hall et al. (6) proposed that uKIM-1 did not predict allograft failure in 91 patients, another 1-year follow-up cohort study led by Szeto et al. (35) showed that uKIM-1 predicted the rate of graft function decline through the detection of messenger RNA (mRNA), which is in accordance with our conclusions. van Timmeren et al. (9) also reported recipients graded by uKIM-1 tertiles as 0.72 (interquartile range, $0.49-1.09$ ) or 1.69 (interquartile range, $1.15-10.04) \mathrm{ng} / 24 \mathrm{~h}$ had a HR of 3.6 (95\% CI, $0.9-13.5)$ or 5.1 (95\% CI, $1.5-17.8)$ for predicting graft loss, respectively. Discrepancies in findings among different studies may be the consequence of diverse cohort population sizes, complication conditions, and the possibility of residual confounding, necessitating further 
A

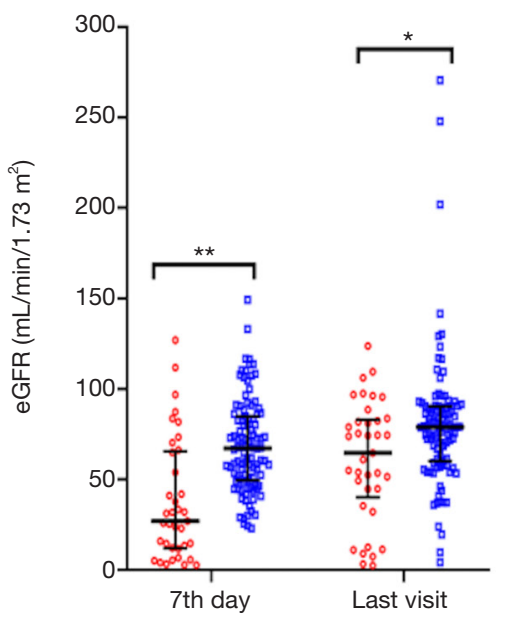

C

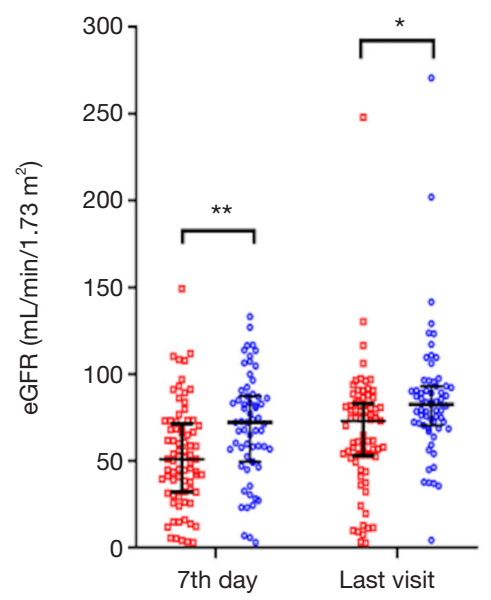

$\mathrm{E}$

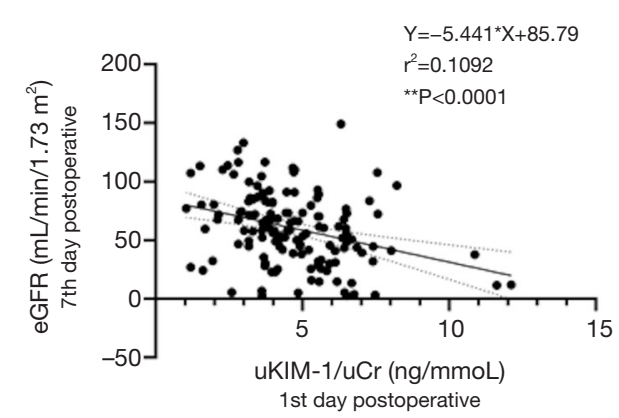

B
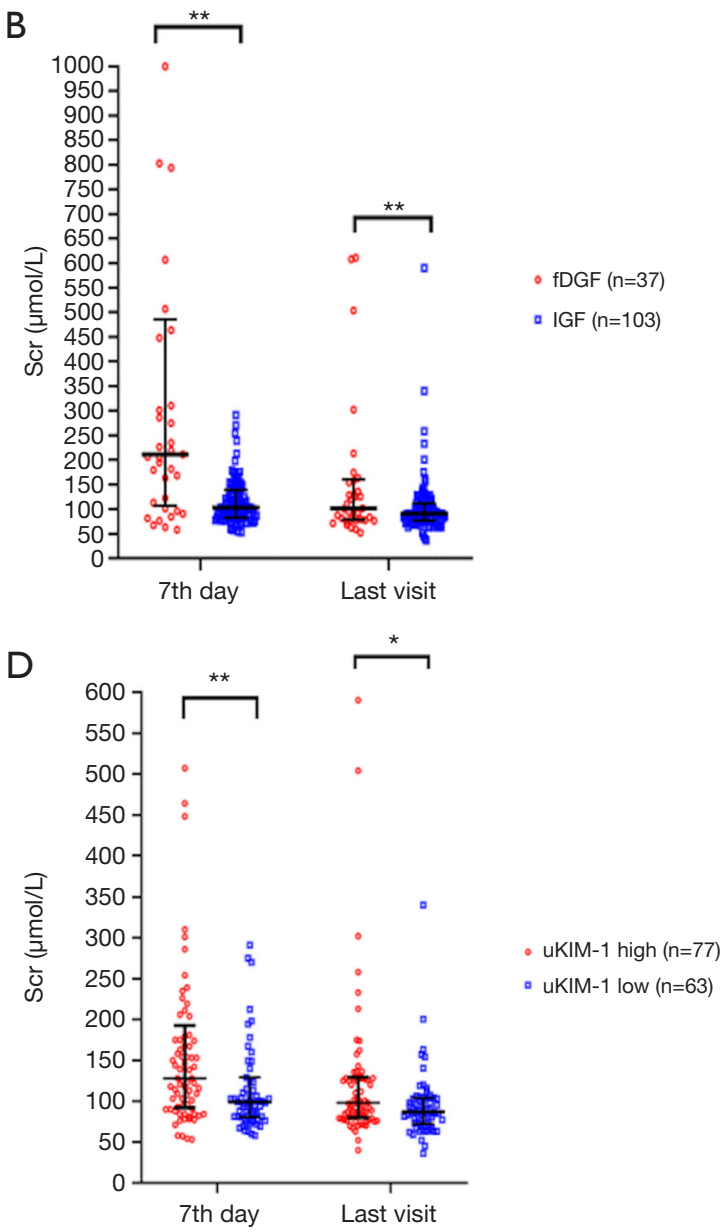

$\mathrm{F}$

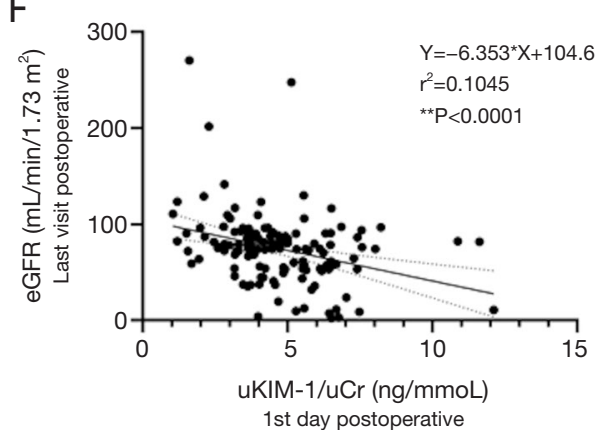

Figure 3 Association between $\mathrm{uKIM}-1 / \mathrm{uCr}$ and graft function at different time points post transplantation. (A,B,C,D) Medians and interquartile ranges for Scr and eGFR after kidney transplantation on day 7 post-surgery and at the last follow-up visit. (E,F) Spearman's correlation analysis between uKIM-1/uCr (1st day post-operation) and $\mathrm{Scr}$ (days 3 and 7 post-surgery). *, $\mathrm{P}<0.05 ;{ }^{* *}, \mathrm{P}<0.01$. fDGF, functional delayed graft function; IGF, immediate graft function; uKIM-1, urinary kidney injury molecular 1; uCr, urinary creatinine; uKIM1/uCr, the ratio of uKIM-1 to uCr; Scr, serum creatinine; uKIM-1/uCr low, uKIM-1/uCr <4.08 ( $\mathrm{pg} / \mathrm{mmol})$; uKIM-1/uCr high, uKIM-1/ $\mathrm{uCr} \geq 4.08$ ( $\mu \mathrm{g} / \mathrm{mmol}) ;$ r, regression coefficient. 


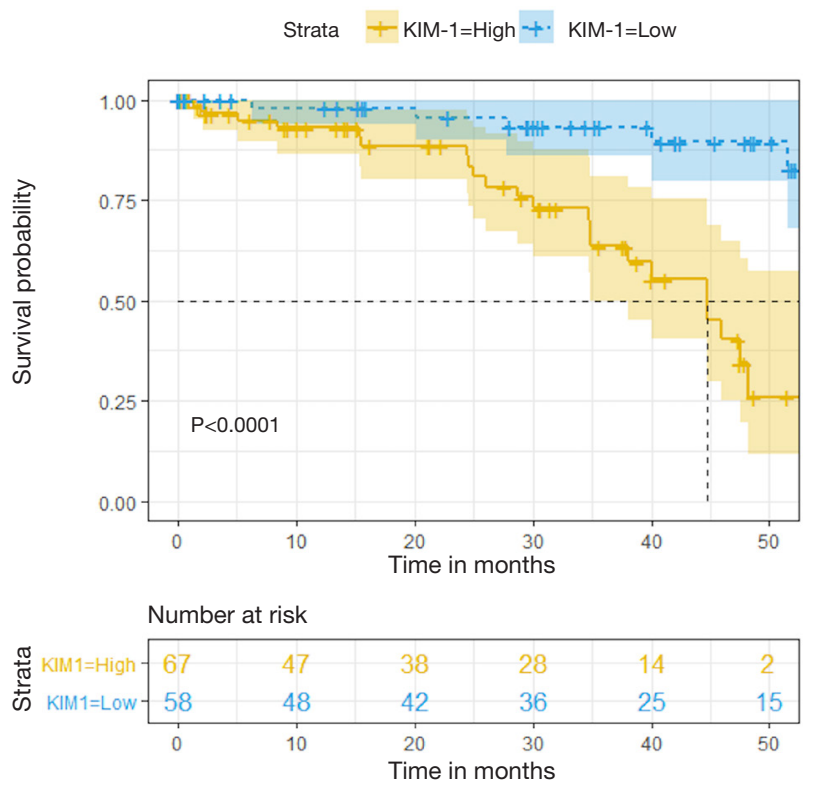

Figure 4 Kaplan-Meier plot of long-term graft dysfunction. Graft dysfunction: eGFR <60 (mL/min/1.73 m²); KIM-1 = low: uKIM-1/uCr $<4.08 \mu \mathrm{g} / \mathrm{mmol}$ (n=58); KIM-1 = high: uKIM-1/uCr $\geq 4.08 \mu \mathrm{g} / \mathrm{mmol}$ (n=67). eGFR, estimated glomerular filtration rate; uKIM-1, urinary kidney injury molecular 1 ; uCr, urinary creatinine.

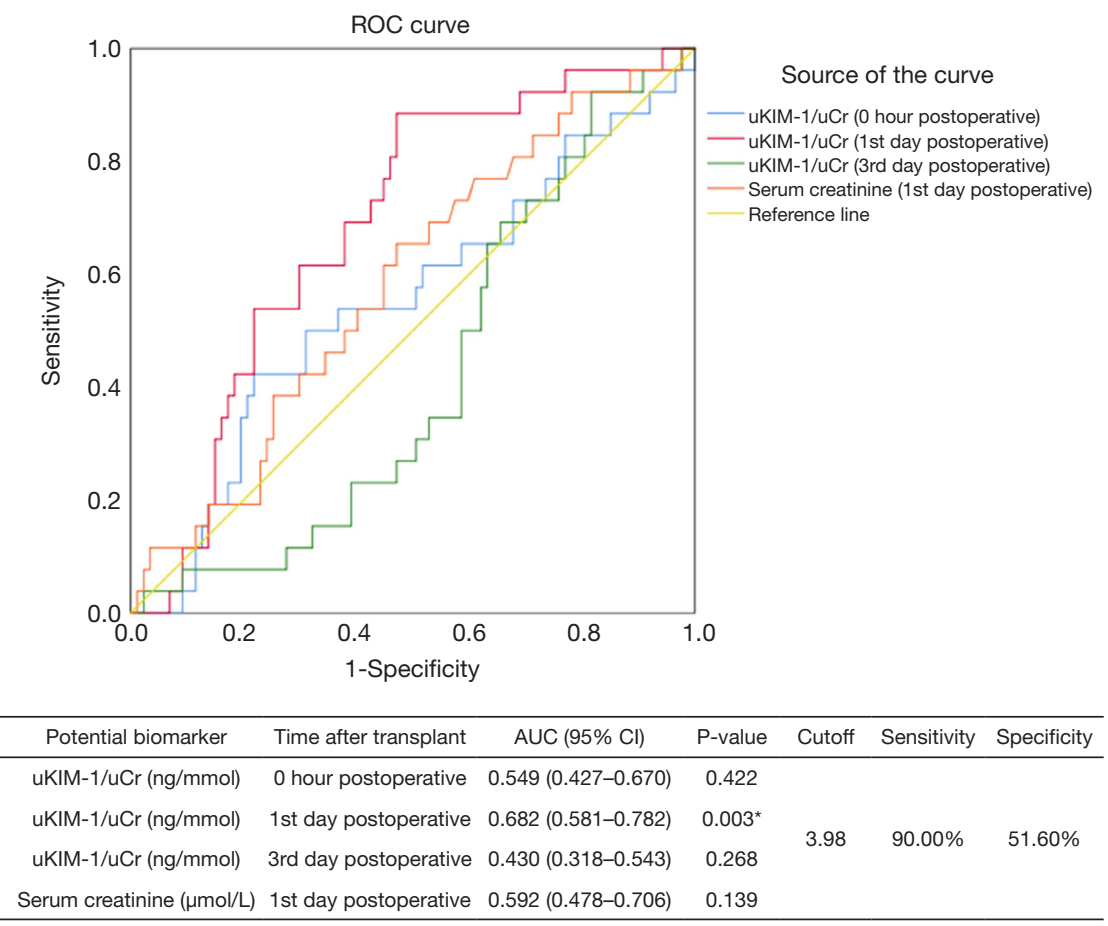

Figure 5 Receiver operating characteristic (ROC) curves and areas under the curves for predicting long-term graft dysfunction. * $\mathrm{P}<0.05$. AUC, area under the curve; 95\% CI, 95\% confidence interval; uKIM-1/uCr, the ratio of uKIM-1 to uCr; uKIM-1, urinary kidney injury molecular 1 ; uCr, urinary creatinine. 


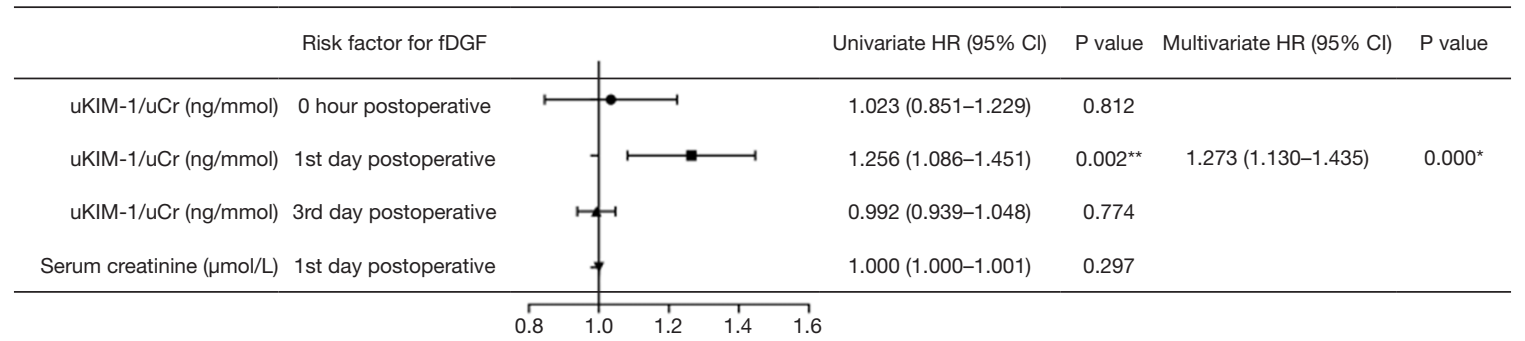

Figure 6 Forest plot of Cox regression analysis in long-term graft function among recipients. * $\mathrm{P}<0.05 ;{ }^{* *}, \mathrm{P}<0.01$. HR, hazard ratio; $95 \%$ CI, $95 \%$ confidence interval; fDGF, functional delayed graft function.

investigation.

Based on the aforementioned literature, our study further detected uKIM-1 at 3 time points after transplantation in a larger cohort of 140 recipients. We demonstrated that on the first day post-surgery, uKIM-1 predicted a $23.5 \%$ increase in the risk of fDGF and a $27.3 \%$ increase in the risk of long-term allograft dysfunction.

Recent studies focusing on DGF have uncovered several promising biomarkers, including NGAL, IL-18, KIM1 , and NAG in particular $(22,24)$. The present study also included L-FABP, a fatty acid-binding protein excreted from the cytoplasm of proximal renal tubular cells in response to acute injury (36,37), NGAL, a siderophore binding protein expressed by distal epithelial cells which is sensitive to AKI $(38,39)$, and NAG, a lysosomal enzyme of proximal tubular cells that is sensitive to proximal tubular injury caused by drugs, environmental toxicants, contrast-induced toxicity, and ischemia $(40,41)$. We found no statistically significant differences between the fDGF group and the IGF group in terms of either NGAL or NAG in the first 3 days post transplantation. Further regression analysis reinforced this result. These findings support those of an American study which indicated that urine NGAL/Cr was independently associated with cardiovascular events rather than fDGF or long-term allograft outcomes (42). Additionally, another report from Nauta et al. (15) confirmed that NAG could not predict graft loss after adjustment with albuminuria, which further validates our conclusion.

Multivariate regression analysis showed that uL-FABP/ Cr on the first day post transplantation was also not related to fDGF. Research by Bansal et al. (42) produced comparable results. Despite being strongly associated with AKI, urinary biomarkers for tubular injury may not be applicable for fDGF or long-term allograft outcomes. A probable explanation may be the slower responses of these biomarkers compared to KIM-1, or there may be a unique mechanism underlying ischemia tubular injury and longterm allograft outcomes that involves KIM-1 but not other markers. Our findings suggest that proximal tubular injury, which is more closely related to fibrosis, contributes to long-term allograft loss, regardless of kidney filtration.

There were also some limitations to this study. Firstly, it is a single-center study. Bias in patient selection might therefore have been introduced. Moreover, extracorporeal membrane oxygenation (ECMO) was not performed during the acquirement of all DCD kidneys in our center. The relationships between uKIM-1, fDGF, allograft loss, and all-cause mortality may be further explored with longer follow-ups in the future. Secondly, our findings require validation in separate cohorts, due to our cohort only covering recipients with a deceased donor kidney. Thirdly, we only tested biomarker levels during the first 3 days after transplantation. Whether uKIM-1 can be restored to a normal level, or if there are other biomarkers of AKI related to allograft outcomes at later time points post-surgery remains uncertain.

\section{Conclusions}

In this cohort of 140 renal transplantation recipients, we found that high uKIM-1 expression on the first day post-surgery predicts short-term fDGF and is a potential biomarker for long-term graft function. This study therefore proposes a potent biomarker for early monitoring and graft deterioration interventions due to graft ischemiareperfusion injury, and a feasible therapeutic target for chronic allograft nephropathy.

\section{Acknowledgments}

We are very grateful to all the medical, nursing, and supportive staff of Department of Nephrology and 
Transplantation Center of Renji Hospital for their dedication in looking after the patients.

Funding: This study was supported in part by Science \& Technology Cooperation Program of China (2017YFE0110500). The study was also sponsored by the National Natural Science Foundation of China (81373865, $81573748,81770668)$ as well as by a grant $(14140903200)$ from the Science and Technology Commission of Shanghai Municipality, China. The study was also sponsored by the Program of Shanghai Academic Research Leader (16XD1401900), a grant $\{[2017] 485\}$ from the Shanghai Leadership Training Program, and a grant (18zxy001) from Shanghai Jiao Tong University School of Medicine.

\section{Footnote}

Reporting Checklist: The authors have completed the STROBE reporting checklist. Available at http://dx.doi. org/10.21037/atm-20-2215a

Data Sharing Statement: Available at http://dx.doi. org/10.21037/atm-20-2215a

Conflicts of Interest: All authors have completed the ICMJE uniform disclosure form (available at http://dx.doi. org/10.21037/atm-20-2215a). The authors have no conflicts of interest to declare.

Ethical Statement: The authors are accountable for all aspects of the work in ensuring that questions related to the accuracy or integrity of any part of the work are appropriately investigated and resolved. The study was conducted in accordance with the Declaration of Helsinki (as revised in 2013). The study was approved by the Ethics Committee of Renji Hospital, School of Medicine, Shanghai Jiao Tong University (14140903200). All participants had signed informed consent.

Open Access Statement: This is an Open Access article distributed in accordance with the Creative Commons Attribution-NonCommercial-NoDerivs 4.0 International License (CC BY-NC-ND 4.0), which permits the noncommercial replication and distribution of the article with the strict proviso that no changes or edits are made and the original work is properly cited (including links to both the formal publication through the relevant DOI and the license). See: https://creativecommons.org/licenses/by-nc-nd/4.0/.

\section{References}

1. Summers DM, Johnson RJ, Hudson A, et al. Effect of donor age and cold storage time on outcome in recipients of kidneys donated after circulatory death in the UK: a cohort study. Lancet 2013;381:727-34.

2. Matas AJ, Smith JM, Skeans MA, et al. OPTN/SRTR 2013 Annual Data Report: kidney. Am J Transplant 2015;15 Suppl 2:1-34.

3. Summers DM, Johnson RJ, Allen J, et al. Analysis of factors that affect outcome after transplantation of kidneys donated after cardiac death in the UK: a cohort study. Lancet 2010;376:1303-11.

4. Chen G, Wang C, Ko DS, et al. Comparison of outcomes of kidney transplantation from donation after brain death, donation after circulatory death, and donation after brain death followed by circulatory death donors. Clin Transplant 2017;31. doi: 10.1111/ctr.13110.

5. Coemans M, Susal C, Dohler B, et al. Analyses of the short- and long-term graft survival after kidney transplantation in Europe between 1986 and 2015. Kidney Int 2018;94:964-73.

6. Hall IE, Yarlagadda SG, Coca SG, et al. IL-18 and urinary NGAL predict dialysis and graft recovery after kidney transplantation. J Am Soc Nephrol 2010;21:189-97.

7. Naesens M, Anglicheau D. Precision Transplant Medicine: Biomarkers to the Rescue. J Am Soc Nephrol 2018;29:24-34.

8. Anglicheau D, Naesens M, Essig M, et al. Establishing Biomarkers in Transplant Medicine: A Critical Review of Current Approaches. Transplantation 2016;100:2024-38.

9. van Timmeren MM, Vaidya VS, van Ree RM, et al. High urinary excretion of kidney injury molecule- 1 is an independent predictor of graft loss in renal transplant recipients. Transplantation 2007;84:1625-30.

10. Schnuelle P, Gottmann U, Hoeger S, et al. Effects of donor pretreatment with dopamine on graft function after kidney transplantation: a randomized controlled trial. JAMA 2009;302:1067-75.

11. Boom H, Mallat MJ, de Fijter JW, et al. Delayed graft function influences renal function, but not survival. Kidney Int 2000;58:859-66.

12. Perico N, Cattaneo D, Sayegh MH, et al. Delayed graft function in kidney transplantation. Lancet 2004;364:1814-27.

13. Pianta TJ, Peake PW, Pickering JW, et al. Clusterin in kidney transplantation: novel biomarkers versus serum creatinine for early prediction of delayed graft function. 
Transplantation 2015;99:171-9.

14. Weiner DE, Park M, Tighiouart H, et al. Albuminuria and Allograft Failure, Cardiovascular Disease Events, and AllCause Death in Stable Kidney Transplant Recipients: A Cohort Analysis of the FAVORIT Trial. Am J Kidney Dis 2019;73:51-61.

15. Nauta FL, Bakker SJ, van Oeveren W, et al. Albuminuria, proteinuria, and novel urine biomarkers as predictors of long-term allograft outcomes in kidney transplant recipients. Am J Kidney Dis 2011;57:733-43.

16. Xie Y, Wang Q, Wang C, et al. High urinary excretion of kidney injury molecule-1 predicts adverse outcomes in acute kidney injury: a case control study. Crit Care 2016;20:286.

17. Levey AS, Coresh J, Greene T, et al. Expressing the Modification of Diet in Renal Disease Study equation for estimating glomerular filtration rate with standardized serum creatinine values. Clin Chem 2007;53:766-72.

18. Marek C, Thomson B, Shoker A, et al. The prognostic value of time needed on dialysis in patients with delayed graft function. Nephrol Dial Transplant 2014;29:203-8.

19. Gonwa TA, Mai ML, Smith LB, et al. Immunosuppression for delayed or slow graft function in primary cadaveric renal transplantation: use of low dose tacrolimus therapy with post-operative administration of anti-CD25 monoclonal antibody. Clin Transplant 2002;16:144-9.

20. Govani MV, Kwon O, Batiuk TD, et al. Creatinine reduction ratio and 24-hour creatinine excretion on posttransplant day two: simple and objective tools to define graft function. J Am Soc Nephrol 2002;13:1645-9.

21. Siedlecki A, Irish W, Brennan DC. Delayed graft function in the kidney transplant. Am J Transplant 2011;11:2279-96.

22. Israni AK, Salkowski N, Gustafson S, et al. New national allocation policy for deceased donor kidneys in the United States and possible effect on patient outcomes. J Am Soc Nephrol 2014;25:1842-8.

23. Tapiawala SN, Tinckam KJ, Cardella CJ, et al. Delayed graft function and the risk for death with a functioning graft. J Am Soc Nephrol 2010;21:153-61.

24. Schröppel B, Legendre C. Delayed kidney graft function: from mechanism to translation. Kidney Int 2014;86:251-8.

25. Shao X, Tian L, Xu W, et al. Diagnostic value of urinary kidney injury molecule 1 for acute kidney injury: a metaanalysis. PLoS One 2014;9:e84131.

26. Xie Y, Xue W, Shao X, et al. Analysis of a urinary biomarker panel for obstructive nephropathy and clinical outcomes. PLoS One 2014;9:e112865.
27. Xu Y, Xie Y, Shao X, et al. L-FABP: A novel biomarker of kidney disease. Clin Chim Acta 2015;445:85-90.

28. Han WK, Bailly V, Abichandani R, et al. Kidney Injury Molecule-1 (KIM-1): a novel biomarker for human renal proximal tubule injury. Kidney Int 2002;62:237-44.

29. Yang L, Brooks CR, Xiao S, et al. KIM-1-mediated phagocytosis reduces acute injury to the kidney. J Clin Invest 2015;125:1620-36.

30. Bonventre JV. Kidney injury molecule-1 (KIM-1): a urinary biomarker and much more. Nephrol Dial Transplant 2009;24:3265-8.

31. Humphreys BD, Xu F, Sabbisetti V, et al. Chronic epithelial kidney injury molecule-1 expression causes murine kidney fibrosis. J Clin Invest 2013;123:4023-35.

32. Desanti De Oliveira B, Xu K, Shen TH, et al. Molecular nephrology: types of acute tubular injury. Nat Rev Nephrol 2019;15:599-612.

33. Vaidya VS, Ferguson MA, Bonventre JV. Biomarkers of acute kidney injury. Annu Rev Pharmacol Toxicol 2008;48:463-93.

34. Xue W, Xie Y, Wang Q, et al. Diagnostic performance of urinary kidney injury molecule-1 and neutrophil gelatinase-associated lipocalin for acute kidney injury in an obstructive nephropathy patient. Nephrology (Carlton) 2014;19:186-94.

35. Szeto CC, Kwan BC, Lai KB, et al. Urinary expression of kidney injury markers in renal transplant recipients. Clin J Am Soc Nephrol 2010;5:2329-37.

36. Noiri E, Doi K, Negishi K, et al. Urinary fatty acidbinding protein 1: an early predictive biomarker of kidney injury. Am J Physiol Renal Physiol 2009;296:F669-79.

37. Kamijo A, Kimura K, Sugaya T, et al. Urinary fatty acidbinding protein as a new clinical marker of the progression of chronic renal disease. J Lab Clin Med 2004;143:23-30.

38. Paragas N, Qiu A, Zhang Q, et al. The Ngal reporter mouse detects the response of the kidney to injury in real time. Nat Med 2011;17:216-22.

39. Haase M, Bellomo R, Devarajan P, et al. Accuracy of neutrophil gelatinase-associated lipocalin (NGAL) in diagnosis and prognosis in acute kidney injury: a systematic review and meta-analysis. Am J Kidney Dis 2009;54:1012-24.

40. Westhuyzen J, Endre ZH, Reece G, et al. Measurement of tubular enzymuria facilitates early detection of acute renal impairment in the intensive care unit. Nephrol Dial Transplant 2003;18:543-51.

41. Kuźniar J, Marchewka Z, Krasnowski R, et al. Enzymuria and low molecular weight protein excretion as the 
differentiating marker of complications in the early post kidney transplantation period. Int Urol Nephrol 2006;38:753-8.

42. Bansal N, Carpenter MA, Weiner DE, et al. Urine Injury
Biomarkers and Risk of Adverse Outcomes in Recipients of Prevalent Kidney Transplants: The Folic Acid for Vascular Outcome Reduction in Transplantation Trial. J Am Soc Nephrol 2016;27:2109-21.

Cite this article as: Zhu M, Chen Z, Wei Y, Yuan Y, Ying L, Zhou H, Che X, Zhang MF, Ni Z, Zhang M, Mou S. The predictive value of urinary kidney injury molecular-1 for long-term graft function in kidney transplant patients: a prospective study. Ann Transl Med 2021;9(3):244. doi: 10.21037/atm-20-2215a 


\section{Supplementary}

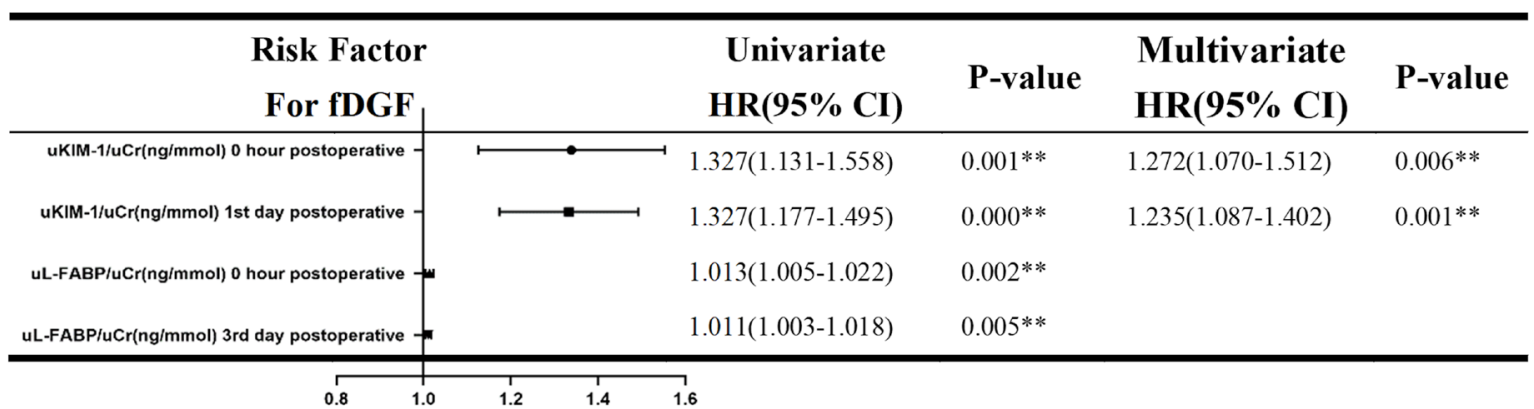

Figure S1 Univariate and multivariate Cox regression analysis in fDGF among recipients. **, $\mathrm{P}<0.01$. HR, hazard ratio; $95 \%$ CI, 95\% confidence interval; fDGF, functional delayed graft function.

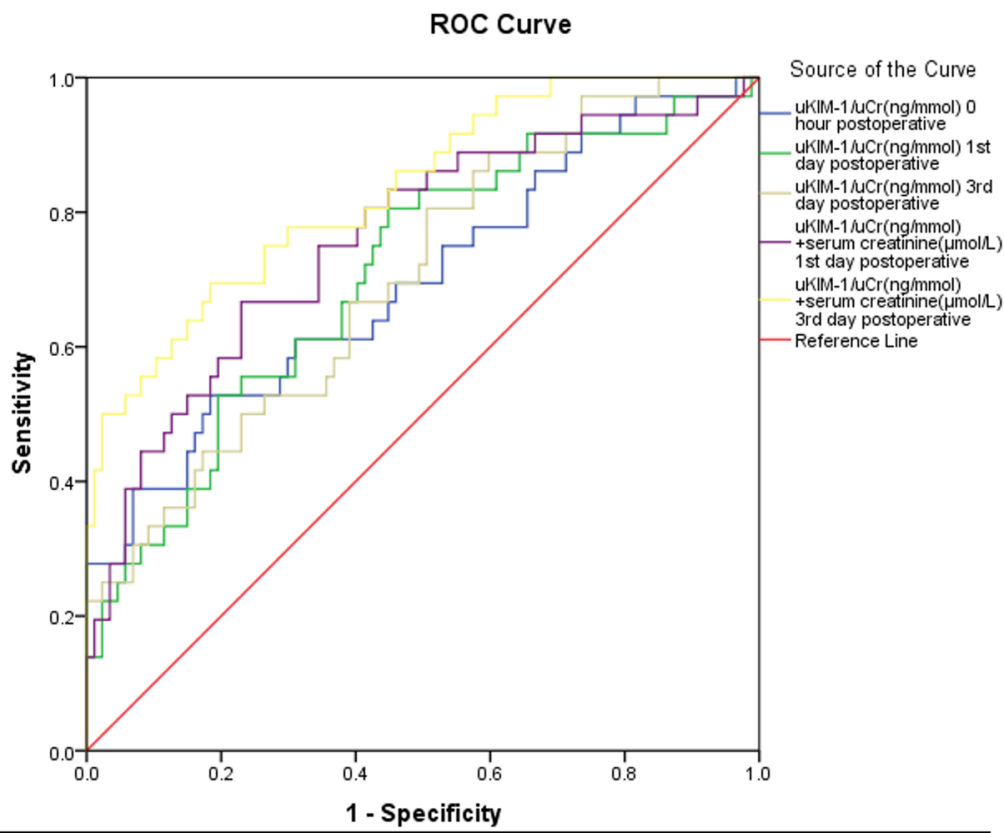

\begin{tabular}{|c|c|c|c|c|c|c|}
\hline Potential Biomarker & $\begin{array}{l}\text { Time after } \\
\text { Transplant }\end{array}$ & AUC(CI 95\%) & p-value & Cutoff & Sensitivity & Specificity \\
\hline (1) $\mathbf{u K I M}-1 / \mathrm{uCr}(\mathrm{ng} / \mathrm{mmol})$ & 0 hour postoperative & $0.684(0.581-0.787)$ & $0.001 * *$ & 5.875 & $51.40 \%$ & $79.60 \%$ \\
\hline (2) uKIM-1/uCr(ng/mmol) & $1^{I^{a}}$ day postoperative & $0.698(0.598-0.799)$ & $0.000 * *$ & 4.077 & $81.10 \%$ & $52.40 \%$ \\
\hline (3) $\mathbf{u K I M}-1 / \mathrm{uCr}(\mathrm{ng} / \mathrm{mmol})$ & $3^{\text {rd }}$ day postoperative & $0.698(0.600-0.797)$ & $0.000^{* *}$ & 12.378 & $78.40 \%$ & $51.60 \%$ \\
\hline (4) Serum creatinine (umol/L) & 0 hour postoperative & $0.533(0.426-0.64)$ & 0.556 & & & \\
\hline (5) Serum creatinine (umol/L) & $1^{s}$ day postoperative & $0.687(0.585-0.789)$ & $0.001 * *$ & 710.75 & $52.80 \%$ & $57.30 \%$ \\
\hline (6) Serum creatinine $(u m o l / L)$ & 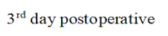 & $0.771(0.674-0.868)$ & $0.000^{* *}$ & 645.00 & $43.20 \%$ & $91.10 \%$ \\
\hline (7) Serum creatinine (umol/L) & $5^{\text {th }}$ day postoperative & $0.761(0.645-0.876)$ & $0.000 * *$ & 208.45 & $63.90 \%$ & $79.80 \%$ \\
\hline (8) Serum creatinine (umol/L) & $7^{\text {th }}$ day postoperative & $0.773(0.664-0.883)$ & $0.000^{* *}$ & 166.30 & $65.60 \%$ & $86.00 \%$ \\
\hline (2) $+(5)$ & $1^{\text {st day postoperative }}$ & $0.761(0.664-0.857)$ & $0.000 * *$ & & & \\
\hline (3) $+(6)$ & $3^{\text {rd }}$ day postoperative & $0.832(0.752-0.911)$ & $0.000 * *$ & & & \\
\hline
\end{tabular}

Figure S2 Receiver operating characteristic curves and areas under the curves for predicting fDGF. *, P<0.05; **, P<0.01. fDGF, functional delayed graft function; ROC, receiver operating characteristic curves; AUC, area under curve; 95\% CI, 95\% confidence interval; uKIM-1, urinary kidney injury molecular 1 ; uCr, urinary creatine; uKIM-1/uCr, the ratio of uKIM-1 to uCr. 
Table S1 Characteristics of donors at baseline

\begin{tabular}{|c|c|c|c|c|}
\hline Characteristics & Total $(n=140)$ & fDGF $(n=37)$ & IGF $(n=103)$ & $P$ value \\
\hline Age & $43.00(30.00-50.00)$ & $48.50(31.75-54.25)$ & $40.00(29.00-50.00)$ & 0.161 \\
\hline Hypertension, n (\%) & $34(24.3)$ & $12(32.4)$ & $22(21.4)$ & 0.178 \\
\hline ICU length of stay (days) & $2.00(1.00-3.00)$ & $2.00(1.00-3.00)$ & $0.00(0.00-00.00)$ & 0.128 \\
\hline Neutrophil (\%) & $83.60(66.55-88.85)$ & 83.65 (46.60-89.65) & 83.10 (77.7-89.10) & 0.871 \\
\hline Lymphocyte (\%) & $10.80(6.75-22.70)$ & $11.50(5.48-43.45)$ & $10.80(7.70-11.70)$ & 0.828 \\
\hline WBC $\left(\times 10^{9} / \mathrm{L}\right)$ & $10.86(5.92-17.64)$ & $11.89(6.23-27.40)$ & $10.86(5.3-15.14)$ & 0.625 \\
\hline UAlb (mg/L) & $10.00(0.00-72.50)$ & $40.00(0.00-93.75)$ & $0.00(0.00-25.00)$ & 0.408 \\
\hline
\end{tabular}

Data that conform to a normal distribution are represented by the mean \pm standard deviation (SD), and non-normally distributed data are represented by the median (interquartile range). Frequency of occurrence of the event is expressed as the frequency (percentage). *, P<0.05. fDGF, functional delayed graft function; IGF, immediate graft function; ICU, intensive care unit; WBC, white blood cells; UAlb, urinary albumin.

Table S2 Cause of recipients' ESRD before kidney transplantation

\begin{tabular}{lccc}
\hline Cause of ESRD & Total $(\mathrm{n}=140)$ & fDGF $(\mathrm{n}=37)$ & IGF $(\mathrm{n}=103)$ \\
\hline GCN & $34(25.60)$ & $13(36.10)$ & $21(21.60)$ \\
IgAN & $28(21.10)$ & $4(11.10)$ & $24(24.70)$ \\
PKD & $4(3.00)$ & $1(2.80)$ & $3(03.10)$ \\
CPN & $3(2.30)$ & $2(5.60)$ & $1(1.00)$ \\
MsPGN & $2(1.50)$ & $1(2.80)$ & $1(1.00)$ \\
Others & $6(4.50)$ & $2(5.60)$ & $4(4.10)$ \\
Unknown & $61(43.60)$ & $14(37.80)$ & $47(45.60)$ \\
HSPN & $2(1.50)$ & $0(0.00)$ & $2(2.10)$ \\
\hline
\end{tabular}

Frequency of occurrence of the event is expressed as the frequency (percentage). ESRD, end-stage renal disease; fDGF, functional delayed graft function; IGF, immediate graft function; GCN, chronic glomerulonephritis; IgAN, IgA nephritis; PKD, polycystic kidney disease; CPN, chronic pyelonephritis; MsPGN, mesangial proliferative glomerulonephritis; Others, include diabetic nephritis, kidney carcinoma, obesity-related glomerulopathy, aristolochic acid nephropathy and thrombotic microangiopathy; HSPN, Henoch-Schŏnlein purpura nephritis.

Table S3 eGFR at last follow-up visit between the fDGF and the IGF group

\begin{tabular}{lccc}
\hline eGFR at last visit $\left(\mathrm{mL} / \mathrm{min} / 1.73 \mathrm{~m}^{2}\right)$ & Total $(\mathrm{n}=140)$ & fDGF $(\mathrm{n}=37)$ & IGF $(\mathrm{n}=103)$ \\
\hline$>90$ & $34(24.30)$ & $7(18.90)$ & $27(26.20)$ \\
$(60,90]$ & $64(45.70)$ & $13(35.10)$ & $51(49.50)$ \\
$(30,60]$ & $31(22.10)$ & $10(27.00)$ & $21(20.40)$ \\
$(15,30]$ & $2(1.40)$ & $0(0.00)$ & $2(1.90)$ \\
$\leq 15$ & $9(6.40)$ & $7(18.90)$ & $2(1.90)$ \\
\hline
\end{tabular}

Frequency of occurrence of the event is expressed as the frequency (percentage). ${ }^{*}, \mathrm{P}<0.05$. eGFR, estimated glomerular filtration rate; fDGF, functional delayed graft function; IGF, immediate graft function. 
Table S4 Comparison of outcomes between the fDGF and the IGF group

\begin{tabular}{lcccc}
\hline Adverse Events & Total $(n=140)$ & fDGF $(n=37)$ & IGF $(n=103)$ & P value \\
\hline Death & $3(2.1)$ & $2(5.4)$ & $1(1.0)$ & 0.110 \\
Graft loss & $9(6.4)$ & $7(18.9)$ & $2(1.9)$ & $0.000^{*}$ \\
\hline
\end{tabular}

Frequency of occurrence of the event is expressed as the frequency (percentage). ${ }^{\star}, \mathrm{P}<0.05$. fDGF, functional delayed graft function; IGF, immediate graft function.

Table S5 Comparison of serum biomarker levels between the fDGF group and the IGF group

\begin{tabular}{|c|c|c|c|c|c|}
\hline Biomarker & Time after transplant & Total $(n=140)$ & fDGF $(n=37)$ & IGF $(n=103)$ & $P$ value \\
\hline \multirow{2}{*}{$\begin{array}{l}\text { sKIM-1 } \\
\text { (ng/L) }\end{array}$} & 1st day postoperative & 80.196 (66.098-105.589) & 73.700 (66.409-97.155) & 87.515 (66.000-110.071) & 0.078 \\
\hline & 3rd day postoperative & 91.075 (70.209-111.417) & $83.270(66.488-98.908)$ & 93.917 (70.713-116.045) & 0.091 \\
\hline \multirow{2}{*}{$\begin{array}{l}\text { sL-FABP } \\
(\mu \mathrm{g} / \mathrm{L})\end{array}$} & 0 hour postoperative & $1,062.729(914.637-1,293.076)$ & $1,059.133(906.646-1,313.145)$ & $1,065.819(914.926-1,293.768)$ & 0.942 \\
\hline & 3rd day postoperative & $1,103.278(917.872-1,301.071)$ & $1,082.246(965.968-1,281.048)$ & $1,110.274(896.099-1,315.960)$ & 0.762 \\
\hline \multirow{3}{*}{$\begin{array}{l}\text { sNGAL } \\
\text { (ng/L) }\end{array}$} & 0 hour postoperative & $8.106(6.965-9.522)$ & $7.542(6.795-9.139)$ & $8.239(7.038-9.570)$ & 0.210 \\
\hline & 1st day postoperative & $7.901(6.987-9.374)$ & 7.595 (6.642-9.348) & $8.033(7.044-9.396)$ & 0.422 \\
\hline & 3rd day postoperative & $8.273(7.041-9.497)$ & $8.000(6.810-9.294)$ & $8.637(7.165-9.564)$ & 0.358 \\
\hline $\begin{array}{l}\text { sNAG } \\
\text { (ng/L) }\end{array}$ & 3rd day postoperative & 217.552 (185.456-262.482) & 209.392 (184.908-252.720) & 222.870 (185.399-265.962) & 0.322 \\
\hline
\end{tabular}

Data that conform to a normal distribution are represented by the mean \pm standard deviation (SD), and non-normally distributed data are represented by the median (interquartile range). fDGF, functional delayed graft function; IGF, immediate graft function; sKIM-1, serum kidney injury molecular 1; sL-FABP, serum L-type fatty acid binding protein; sNGAL, serum neutrophil gelatinase-related lipoprotein; sNAG, serum $N$-acetyl- $\beta$-D glucosaminidase.

Table S6 Spearman's correlation coefficients between urinary biomarkers and traditional serum creatine at different timepoints post transplantation

\begin{tabular}{|c|c|c|c|c|c|c|c|}
\hline Biomarker & Time after transplant & $\begin{array}{c}\text { uKIM-1/uCr } \\
\text { (0 hour } \\
\text { postoperative) }\end{array}$ & $\begin{array}{c}\text { uKIM-1/uCr } \\
\text { (1st day } \\
\text { postoperative) }\end{array}$ & $\begin{array}{c}\text { uKIM-1/uCr } \\
\text { (3rd day } \\
\text { postoperative) }\end{array}$ & $\begin{array}{c}\text { uL-FABP/uCr } \\
(0 \text { hour } \\
\text { postoperative) }\end{array}$ & $\begin{array}{c}\text { uL-FABP/uCr } \\
\text { (1st day } \\
\text { postoperative) }\end{array}$ & $\begin{array}{c}\text { uL-FABP/uCr } \\
\text { (3rd day } \\
\text { postoperative) }\end{array}$ \\
\hline \multirow{3}{*}{$\begin{array}{l}\text { Serum } \\
\text { creatinine } \\
(\mu \mathrm{mol} / \mathrm{L})\end{array}$} & 1st day postoperative & 0.112 & 0.128 & $0.185^{\star}$ & 0.054 & 0.099 & 0.115 \\
\hline & 3rd day postoperative & 0.052 & $0.256^{\star}$ & 0.119 & 0.031 & 0.099 & 0.072 \\
\hline & 7th day postoperative & 0.158 & $0.335^{\star \star}$ & 0.172 & 0.128 & 0.155 & 0.130 \\
\hline
\end{tabular}

${ }^{*} \mathrm{P}<0.05$; ${ }^{*} \mathrm{P}<0.001$. UKIM-1, urinary kidney injury molecular $1 ; \mathrm{uCr}$, urinary creatinine; uL-FABP, urinary L-type fatty acid binding protein; $\mathrm{uKIM}-1 / \mathrm{uCr}$, the ratio of $\mathrm{uKIM}-1$ to $\mathrm{uCr}$; $\mathrm{uL}-\mathrm{FABP} / \mathrm{uCr}$, the ratio of $\mathrm{uL}-\mathrm{FABP}$ to $\mathrm{uCr}$. 